\title{
Design and hard-in-loop simulation of the real time flight safety monitoring based on ADS-B
}

\author{
LIU Yichao ${ }^{\mathrm{a}}$, WANG Qiang ${ }^{\mathrm{b}}, \mathrm{SHI}$ Hong $^{\mathrm{c}}$ \\ College of Equipment Management \& Safety Engineering, Air Force Engineering University, \\ Xi'an, China \\ a237369687@qq.com, bwq01010004@126.com icy112357515@163.com
}

Keywords: ADS-B; human factors; flight safety; real time monitoring; hard-in-loop simulation

\begin{abstract}
The real time and continuity flight safety monitoring based on ADS-B raised to solve the ACARS report sparse uneven and time delay.The structure of real time flight safety monitoring based on ADS-B is proposed firstly, which monitor the human factors during flight. Then in the concept of practicality and reliablity, an integrative simulate platform for flight safety monitoring is established with current cyber-assistant software. Based on real time flight safety monitoring architecture and simulate platform, the normative flow for the design of flight safety monitoring is set up.
\end{abstract}

\section{Introduction}

According to data, $70 \%$ of air accidents are caused by human factors [1,2]; therefore, it is inevitable that researches on the human factor of pilots become an important link to aviation safety. Researches on human factors of aviation safety focus on following five aspects [3] including track check, LOSA, accident investigation, ADREP and FOQA; among which, the track check and the LOSA are taken advantage of by experts to evaluate flight performance and risk management ability of the aircrew during flying. While another three are utilized to summarize historical accidents and transfinite operations in the process of flying to find out innate factor affecting aviation safety [4-5]. For FOQA, it adopts the ACARS Air-Ground Data Link to monitor essential flight parameters, such as the liftoff speed at the takeoff phase, that influence the aviation safety [3]. Nevertheless, as ACARS messages with uneven distribution of track points are scattered and few in number, their completeness needs to be enhanced further. In addition, its time delay which ranges from 5 to 20 seconds approximately is far from meeting demands of dangerous operation second level detection and early warning.

ADS-B data link of Mode $\mathrm{S}$ is independent of ground radar network and has strong real-time information transmission ability. In this paper, it is discussed that how to utilize the ADS-B data link of Mode $S$ to fulfil real-time and uninterrupted aviation safety monitoring during the entire flying; besides, combining the simulation and inverse simulation of flight dynamics, mal-operations taking place in the process of flight are detected and warned about in real time.

\section{Real-time Aviation Safety Monitoring Based on ADS-B}

ADS-B transmitter of an airplane spontaneously broadcasts ADS-B messages at a certain period dispensing with responses or enquiries. Contents of those messages incorporate the three-dimensional position, three-dimensional velocity, the current intent information and airway intent information, etc. [6] which are all mainly adopted to improve flight safety at present by means of two measures. One is the guarantee of minimum flight interval and the other is the conflict avoidance and resolution. Comparing with the ACARS message, it has an extremely favourable real-time performance. In addition to updating information of position and velocity every 0.4 to 0.6 seconds, broadcasting of any required information can be realized through software and hardware allocations [6]. In this part, the application of ADS-B technology into the real-time monitoring of aviation safety is discussed to 
establish a real-time aviation safety monitoring system as shown in Fig 1.

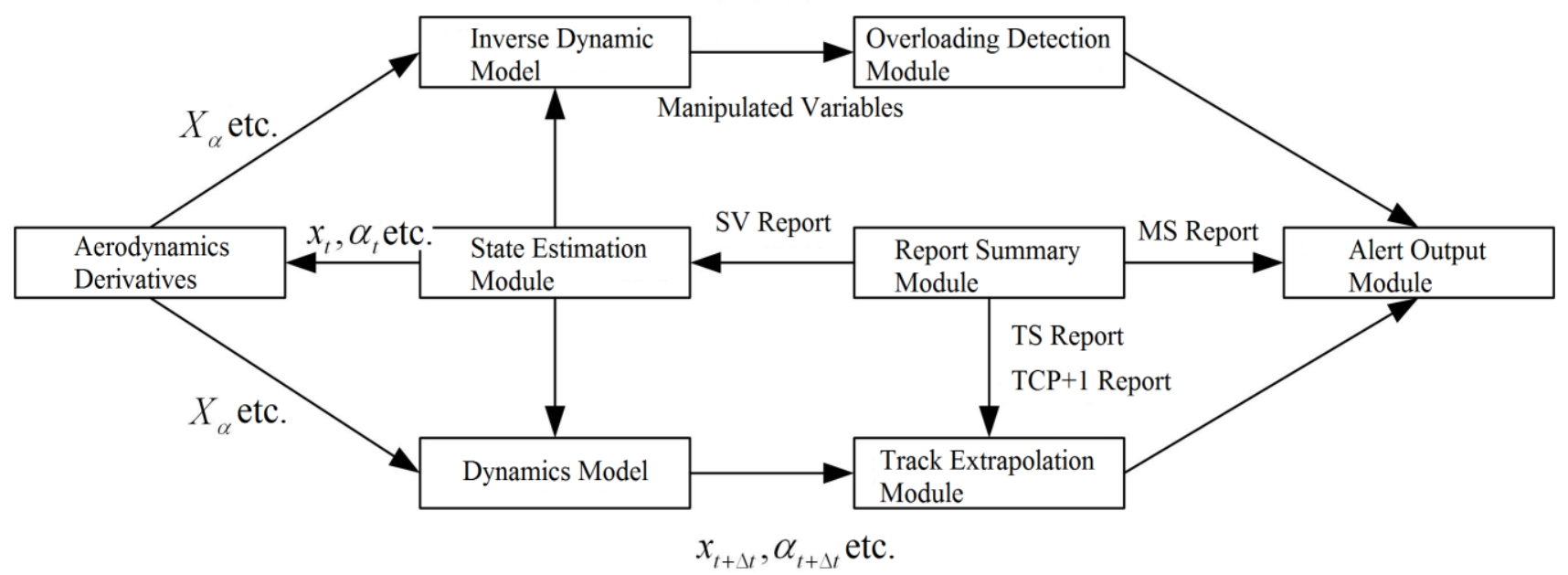

Fig 1. Configuration of the Real-time Monitoring System Based on ADS-B

In the figure above, functions of each module can be described as follows.

The report summary module provides 5 kinds of ADS-B reports [6] that can be directly used by other modules, such as state vector (SV) report, model state (MS) report, target state (TS) report, airspeed reference (ARV) report and the next track change point $(\mathrm{TCP}+1)$ report.

In accordance with three-dimensional position, three-dimensional velocity and roll angle, etc., the state estimation module estimates state variables including $x, y, z, \phi, \theta, \varphi, p, q, r, V, \alpha, \beta$ required by spacecraft dynamics modeling.

Both aerodynamic forces and pneumatic torque derivatives of all state points within the entire flight envelope are stored in the aerodynamics derivative module which carries out retrieval and interpolation as well as outputs aerodynamic derivatives needed by modeling in line with the current state of the airplane.

The inverse dynamic model calculates current accelerator and control surface inputs of $\delta_{T}, \delta_{a}, \delta_{e}, \delta_{r}$ based on the state of airplane.

The dynamics Model calculates the state changing course of airplane within a certain period according to the accelerator and control surface inputs.

The track extrapolation module controls the dynamics Model to calculate the track of air vehicle within the time of $\Delta t$ according to the current state of airplane, the current control of pilot and the intent information report.

The overloading detection system evaluates manipulated variables for the pilot in accordance with flight manual.

The alert output module detects the pilot operation intention and track tendencies to judge possible dangerous consequences such as heavy landing, stall and spiral, etc. caused by mal-operations and make a warning.

\section{Semi-physical Testing Platform}

\subsection{Overall Plan}

According to the statistics of America, the test cycle can be shortened by $25 \%-35 \%$ and the number of test flights can also be reduced by $33.8 \%$ after the adoption of semi-physical simulation technology [7]. As a result, the semi-physical simulation scheme is employed in this paper to perform real-time simulations for the dynamics calculating of air vehicles and the air-ground data transmission 
link to improve both the confidence coefficient and the reliability of such simulations.

Based on the principle of being practical and efficient, existing engineering auxiliary tools are utilized to construct a simulation experiment platform as shown in Fig 2. This platform can be used to test the air-ground data transmission performance, set up a semi-physical simulation testing platform of designated type through a normalized, engineered and standardized procedure and detect the validity of human factor detection algorithm for dangerous operations and transfinite events, etc..

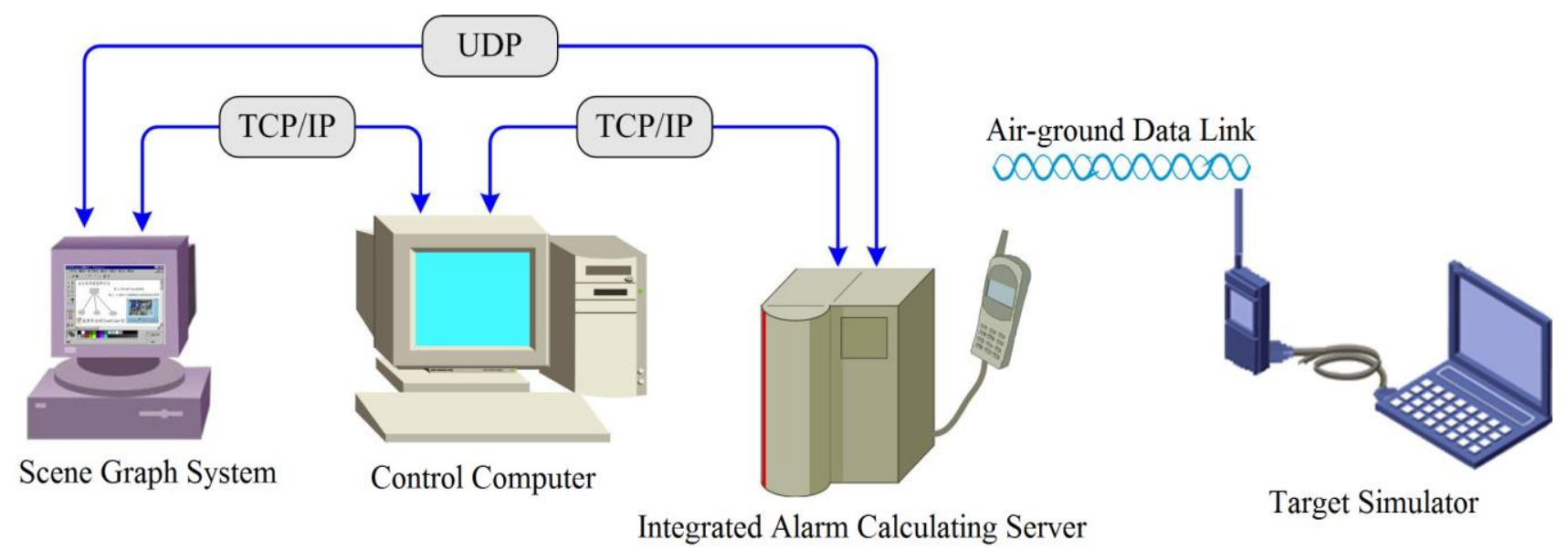

Fig 2. Schematic Diagram of Semi-physical Testing Platform

The simulation test environment is composed of four parts: a target simulator, an integrated alarm calculating server, a scene graph system and a control computer.

To be specific, the target simulator randomly utilizes QAR historical data to simulate the movement state of designated aerial targets so as to provide their position and status information. After that, broadcasting is conducted for the target state through ADS-B in accordance with the protocol coding of extended Mode S (1090Mhz ES).

The calculating server performs transfinite detection combined with the state data by calculating the manipulated variable of pilots based on the inverse simulation on one hand; and on the other hand, it accords with the current manipulated variables of pilot and state data to perform flight path extrapolation in line with which intention assessment and action prediction are conducted and potential insecure factors are warned about, so as to prevent the airplane from getting into a dangerous state of spiral and stall, etc..

The control computer can be employed to modify parameters online and control operations of calculating server and scene graph system during real-time simulations, etc.. Centering on Matlab/Simulink, modeling starts firstly in the environment of Simulink followed by simulation analysis in Matlab. In case that relatively satisfactory simulation results are achieved, they are downloaded to the calculating server for real-time simulation after compiling in RTW.

The scene graph system adopts the open-sourcing FlighGear software. Serving as the main visual window of such a platform, it is not only equipped with a powerful and real flight simulation function, but has a flexible external output/input interface that is able to read flight state parameters from the calculating server directly[8]. Meanwhile, warning messages given by this system in accordance with the calculating server can be output with superposition.

\subsection{Aerial Target Simulator}

Key data contained in the ADS-B message consist of the 24-bit ICAO address, the speed, the longitude, the latitude, the altitude, the heading and the plane identity (flight number), etc.. In the process of flying, ICAO and flight number of the target remain unchanged while its speed, longitude, latitude and altitude, etc. vary in real time together with changes in its movement state. Consequently, 
the simulation should be carried out in line with different dynamic models of the target.

Calculating the kinetic equation is the most important method to simulate the movement target [9]; however, as it is highly dependent of the model, corresponding data cannot be used to examine the accuracy of model and the validity of platform in the follow-up work. In this part, QAR historical data are employed to simulate the movement target. First, air line is cut based on the phase of flight to establish a historical aerial works library; then, historical aerial works are selected in a random manner to perform permutation and combination so as to generate a complete and reasonable line of flight.

\section{(1) Air Line Cutting}

A complete flight can be divided into 5 phases including ground sliding, climbing, cruising, descending and landing, as given in Fig 3.

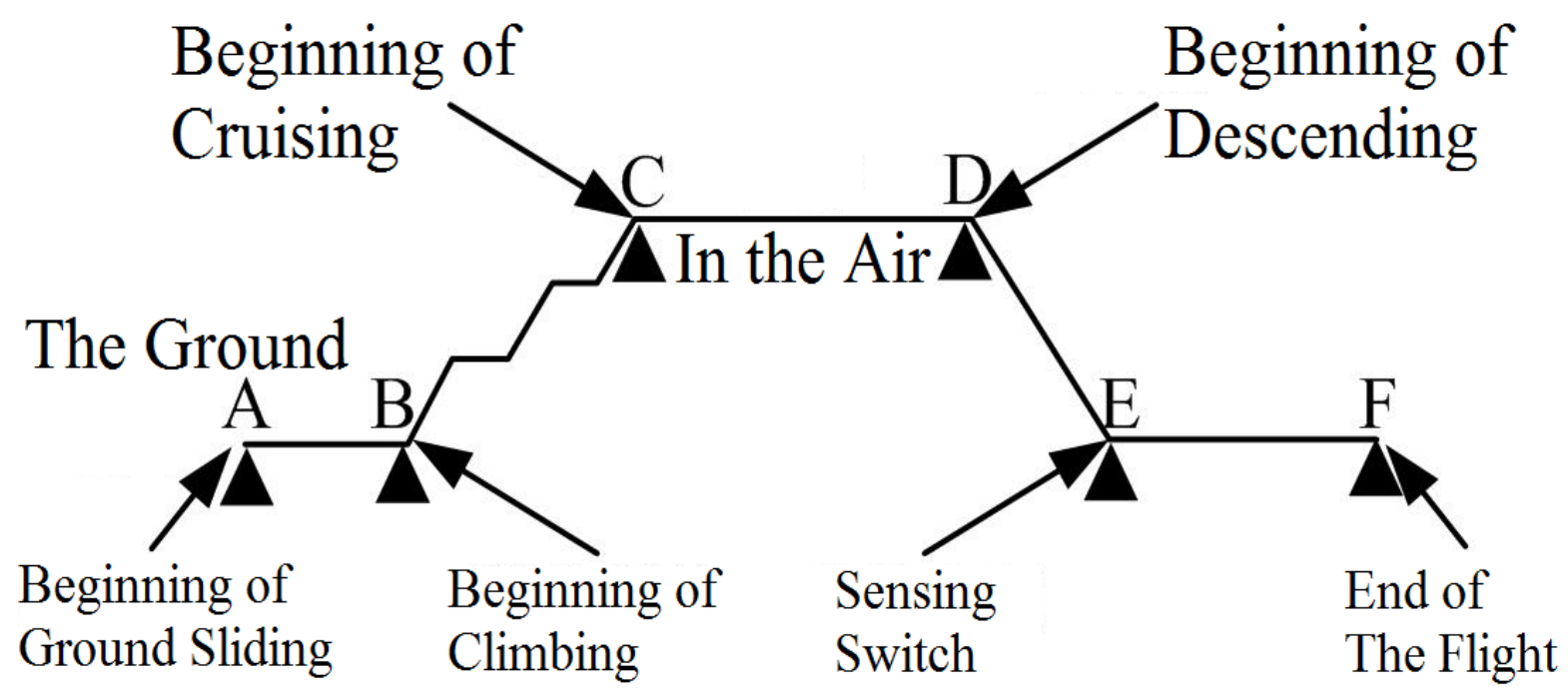

Fig 3. Schematic Diagram of Air Line Cutting

Among them, the phase of climbing refers to the stepped climbing constituted by multiple level flights and climbing sections; and the cruising phase represents winding cruising composed of level flights and curve sections. In this part, sorted storing is performed for each air line according to criteria with an aim to set up a library of 6 historical aerial works such as ground sliding, level flight, climbing, turning, descending and landing. 
Table I. Table of Action Library Establishment Criteria

\begin{tabular}{|c|c|c|c|}
\hline Serial No. & Flight Phase & Aerial Works & Criteria \\
\hline 1 & Ground Sliding & Ground Sliding Beginning Point A & Jumping of Flight Parameter Frame Number \\
\hline 2 & \multirow{3}{*}{ Climbing } & Climbing Beginning Point B & " 1 " is set for the sensing switch for 7 times successively. \\
\hline 3 & & Level Flight & $|\dot{h}| \leq 5 \mathrm{~m} / \mathrm{s}$ for 10 times successively \\
\hline 4 & & Climbing & $|\dot{h}|>5 \mathrm{~m} / \mathrm{s}$ for 10 times successively \\
\hline 5 & \multirow{3}{*}{ Cruising } & Cruising Beginning Point $\mathrm{C}$ & $h \geq \bar{h}$ \\
\hline 6 & & Level Flight & $|\dot{\psi}| \leq 1^{\circ} / s$ for 10 times successively \\
\hline 7 & & Turning & $|\dot{\psi}|>1^{\circ} / s$ for 10 times successively \\
\hline 8 & Descending & Descending Beginning Point $\mathrm{D}$ & $h \leq h_{0}$ \\
\hline 9 & \multirow{2}{*}{ Landing } & Landing Beginning Point $\mathrm{E}$ & " 0 " is set for the sensing switch. \\
\hline 10 & & Landing Ending Point $\mathrm{F}$ & Pushed straight after point $E$ until the jumping of framing counting. \\
\hline
\end{tabular}

In the table, $\mathrm{h}=-\mathrm{z}$ is altitude while $\bar{h}$ the average cruising altitude and $\psi$ the yaw angle.

(2) Air Line Restructuring

A complete target airline is constituted by historical aerial works that are randomly selected from historical aerial works libraries. Any two aerial works are able to be joined and combined provided that the first action ending point $T_{E i}$ and the second action beginning point $T_{S(i+1)}$ have the same state and state variation trends; that is, the aerodynamic force giving rise to such a variation tendency is consistent with the moment of force.

Fig 4 displays a part of the Xi'an-Beijing flight linked and combined by taking one historical aerial work from flights of Chengdu-Lhasa and Chengdu-Beijing. 


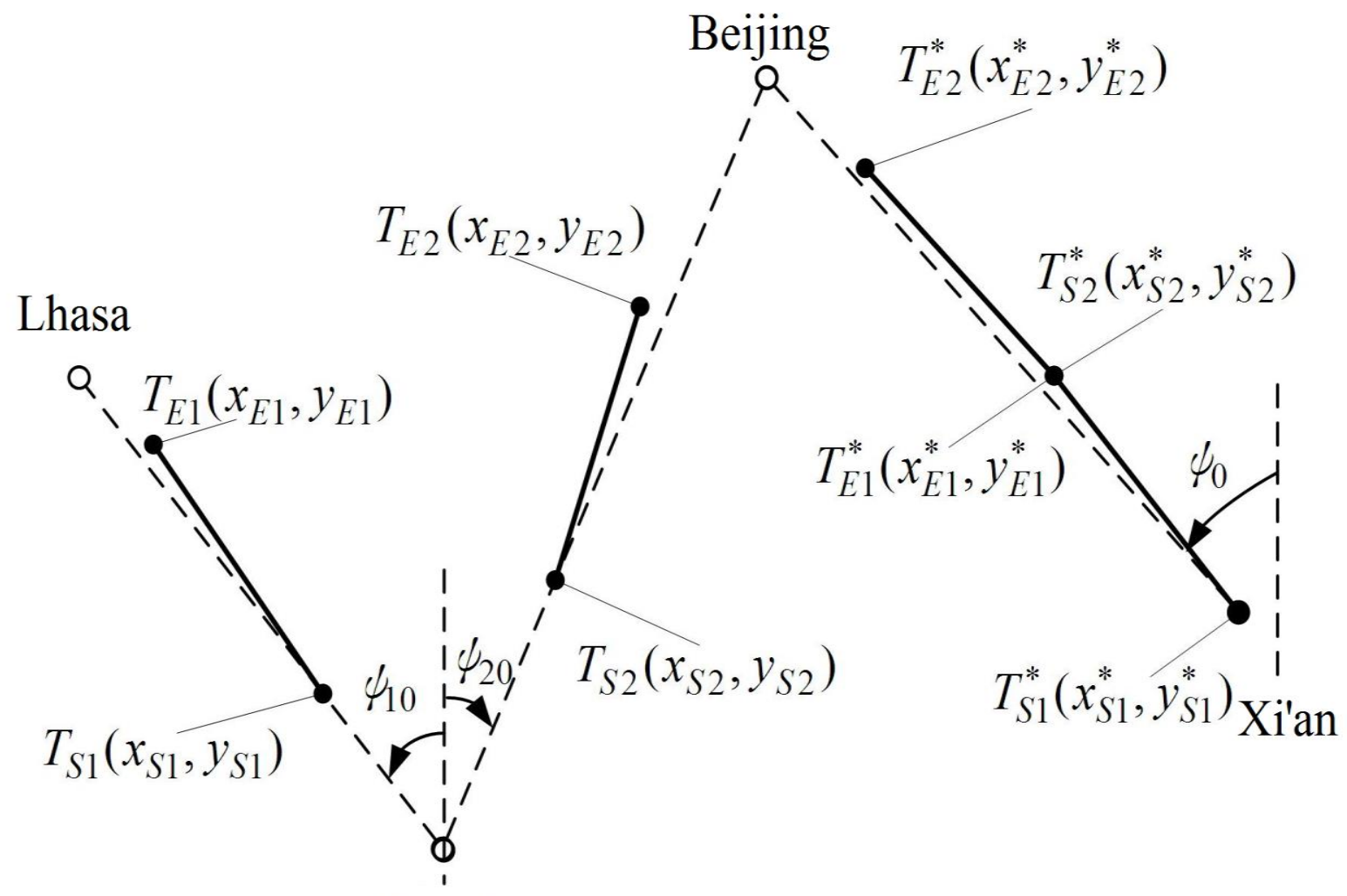

\section{Chengdu}

Fig 4. Schematic Diagram of Air Line Restructuring

In this figure, $T_{S 1}-T_{E 1}$ denotes the ground sliding section of Chengdu-Lhasa flight, while $T_{S 2}-T_{E 2}$ is the climbing section of Chengdu-Beijing flight, $\psi_{0}$ is the course angle between airports of departure and landing for the target flight, $\psi_{i 0}$ is the course angle between airports of departure and landing for the $\mathrm{i}$ historical flight. Their connection \& combination methods are presented below.

(1) Longitude, latitude and heading in $T_{S 1}-T_{E 1}$ are rewritten as $x, y,\left(\psi-\psi_{10}+\psi_{0}\right)$; after the adjustment, $T_{S 1}^{*}-T_{E 1}^{*}$ begins at Xi'an Xianyang International Airport and the included angle between it and Xi'an-Beijing airline is equal to that formed by $T_{S 1}-T_{E 1}$ and the air line of Chengdu-Lhasa.

(2) Similarly, Longitude, latitude and heading in $T_{S 2}-T_{E 2}$ are rewritten $\operatorname{into}\left(x-x_{E 1}^{*}\right),\left(y-y_{E 1}^{*}\right),\left(\psi-\psi_{20}+\psi_{0}\right)$.

(3) On the premise that the error of state variables $z, \phi, \theta, p, q, r, V, \alpha, \beta$ of $T_{E 1}^{*}\left(x_{E 1}^{*}, y_{E 1}^{*}\right)$ and $T_{S 2}^{*}\left(x_{S 2}^{*}, y_{S 2}^{*}\right)$ is more than $5 \%$, two air lines can be linked and combined into one; otherwise, historical aerial works should be selected over again.

\subsection{Transmit-receive System}

(1) Transmission Sub-system

The transmission sub-system is formed by a RF front-end transmitting module and an encoder; hardware construction of the latter is FPGA+ARM, as shown in Fig 5. 


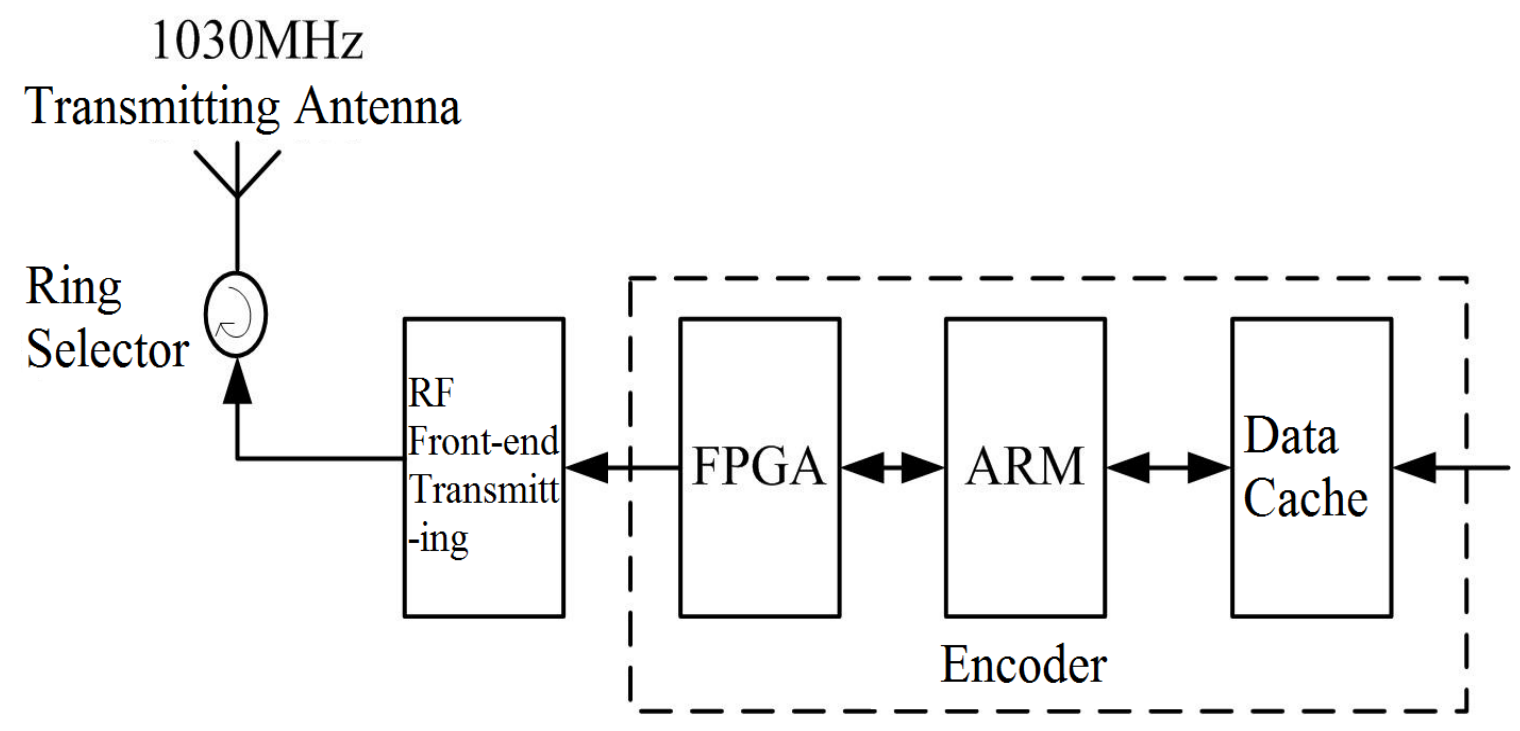

Fig 5. Block Diagram of ADS-B Transmission Sub-system

ARM module mainly takes the responsibility to receive target state data sent by the target simulator and deliver the information contents that are extracted and calculated based on those data in accordance with replacement rates of various information to the FPGA module. After receives such information, the FPGA module calculates and fills check bit PI for all target information; moreover, with the addition of fixed message header, those PIs are sent to the RF front-end transmitting module that modulates and magnifies digital signals sent over by the FPGA module. In the end, those signals can be transmitted at a frequency of $1,090 \mathrm{MHz}$.

\section{(2) Receiving Sub-system}

The ADS-B data link of Mode S has a simple system message format and a relatively weak information carrying capacity. Therefore, the information of one specific type can be transmitted in one encoder. In addition, not only are replacement rates of such information different from each other, but the information of both position and speed is updated every 0.4 to 0.6 seconds while each update of identification and type information as well as that of the intention variation information take place every 4.8-5.2 and 1.6-1.8 seconds respectively [10]. The receiving sub-system includes a demodulator and a report assembler module, as shown in Fig 6.

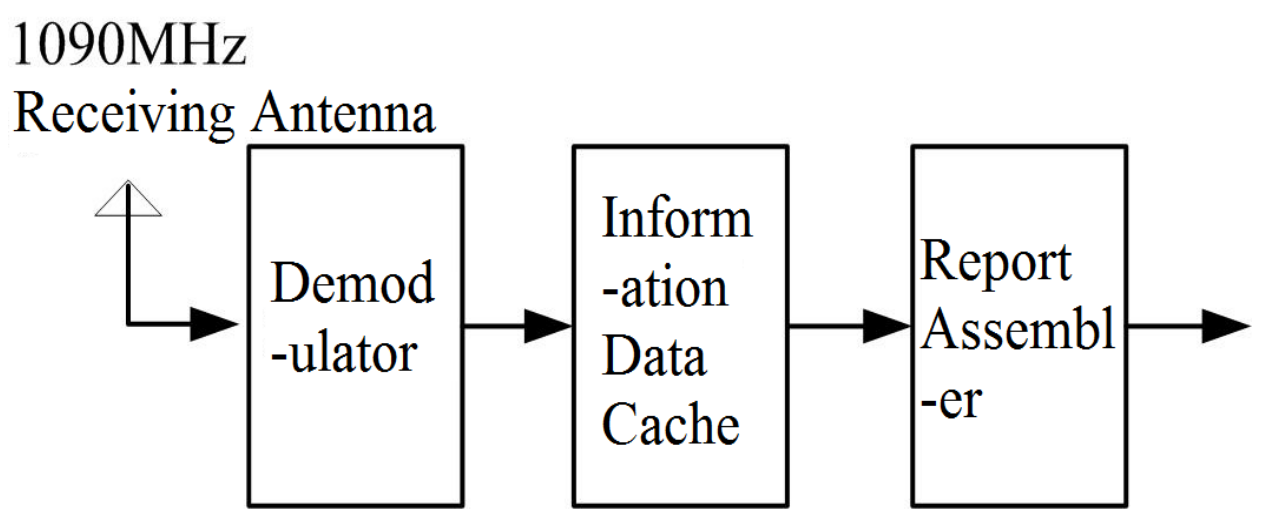

Fig 6. Block Diagram of Receiving Sub-system

Concerning the demodulator, it is provided with a function of information reception in addition to which it is also able to detect the extended interrupted oscillation of external Mode $\mathrm{S}$ and convert it 
into information. With respect of the report assembler module, it collects and decodes dispersed ADS-B information that will be compiled into Category V ADS-B report used by other airborne equipment [6] and output to the data bus connected with applications.

\subsection{Dynamics Modelling for Air Vehicles}

\section{(1) Aerodynamic Modelling}

As for the aerodynamic Modelling, engineering estimation method is adopted; moreover, the aerodynamic computation uses DATCOM and software PropCalc is utilized to perform engine modelling, etc. [11]. The engineering estimation method takes advantage of experimental data acquired through several decades to obtain aerodynamic and operational stability parameters of the air vehicles in a rapid manner. It not only can be used both conveniently and efficiently, but has a very strong adaptability; especially for subsonic flights, its computation accuracy can be up to $90 \%$ and above by precise geometric profile modelling [12].

\section{(2) Dynamics Model}

Flight dynamics Model is base and core of flight simulation and it mainly consists of a flight dynamics Model, a steering engine model, an environmental model and a sensor model, etc.. The module library based on MATLAB/Simulink environment includes the Aerospace set, the AeroSim set and FDC. Integration of basic modules such as engine body dynamics module, propulsion module, steering engine module, aerodynamic force module and atmospheric environment module is fulfilled by them, so is the integration of interface between the geographic model (horizontal reference surface; gravitational field; geomagnetic field, etc.) and the Microsoft Flight Simulator and FlightGear [8].

\section{(3) Inverse Dynamics Model}

The inverse dynamics Model takes no account of big maneuvering flights about passenger aircrafts and military transport aircrafts. It is completely decoupled longitudinally and laterally and inversion of its linear small perturbation equation is carried out directly at each state point.

\section{Engineering Assessment Procedure}

On this testing platform, design, simulation and verification of real-time air safety monitoring are fulfilled in accordance with the following engineering procedures.

Step1. Measure geometric data of the airplane and compile DATCOM input file to conduct aerodynamic estimation; aircrafts;

Step2. Use Aerospace to establish the dynamics Model for the six degrees of freedom of

Step3. Inspect the validity of dynamics Model; if valid, proceed to the next step; if not, return back to the first step;

Step4. Set up the inverse simulation dynamics Model in line with the dynamics Model;

Step5. Inspect the validity of such an inverse simulation dynamics Model according to aircraft configuration and manipulated variables; if valid, proceed to the next step; if not, proceed to Step 4;

Step6. Calculate the current manipulated variables by means of the inverse simulation model according to the current state provided by ADS-B messages;

Step7. Perform pilot transfinite analysis based on aircraft configuration and manipulated variables;

Step8. Perform flight path extrapolation, action identification and intention recognition through dynamics simulation in line with the aircraft configuration and the manipulated variable obtained by 
inverse simulation calculating;

Step9. Check and simulate the flight time history of the aircraft entering a hazardous situation and predict remaining time and changing approaches.

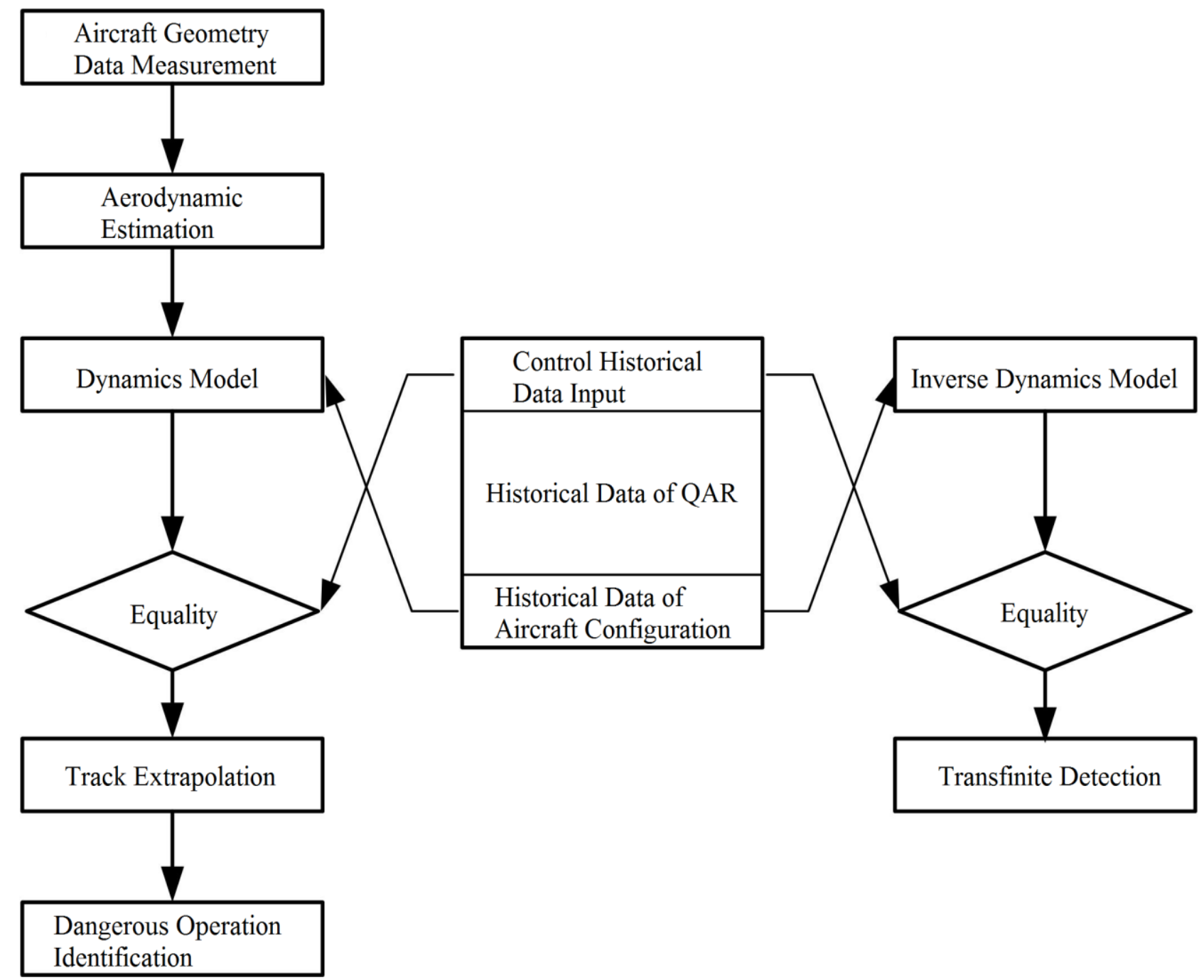

Fig 7. Engineering Assessment Procedure

\section{Conclusions}

It is put forward in this paper that real-time and uninterrupted air safety monitoring can be realized based on ADS-B technology and it is able to detect and warn about mal-operations existing during the flight in real time. In addition, an ADS-B real-time monitoring architecture of air safety is also designed; moreover, a semi-physical testing platform and set of engineering and standard procedures related to the real-time air safety monitoring system are also established. As a result, problems such as spare messages with a weak real-time performance existing in current real-time monitoring are solved so as to promote functionization and engineering process for real-time air safety monitoring effectively.

\section{References}

[1] Sun Ruishan; Wang Xin. Application of CREAM Failure Probability Prediction Method in Aircrew Judgment and Decision-making for Cockpit [J]. Journal of Safety Science and Technology, 2010, 6(6): 40-45. 
[2] Xu Bailing. Lessons Drawn from Others' Mistakes - Review and Reflections on Civil Aviation Flight Safety of New China. Beijing: China Civil Aviation Press. 1999: 36-35.

[3] Wang Tianming. Research on Flight Safety Model based on QAR Data [D]. Civil Aviation University of China, 2008.

[4] Wiegmann D A; Shappell S. A.. A Human Error Analysis of Commercial Aviation Accidents Using the Human Factors Analysis and Classification System[R]. DOT / FAA / AM-01 / 3, 2001.

[5] Lang Guopeng. Analysis of Human Errors in Aviation Safety Management and Its Countermeasures [J]. Journal of Civil Aviation University of China, 2009, 27(4): 18-21.

[6] Yao Jiao. A Performance Study and Simulation of ADS-B Surveillance Function [D]. Chengdu: University of Electronic Science and Technology of China, 2010.

[7] Liu Liangliang; Hu Yanlin; Yi Mu et al.. Research on Semi-physical Simulation System of UAV [J]. Advanced Manufacturing and Management, 2008, 27(3): 44-45.

[8] Curtis. L. Olson. Flight Gear is an Open-source Flight Simulator [OL/DB]. http://www.flightgear.org/

[9] Hu Chenbo. Design and Fulfillment Based on ADS-B Multi-target Simulator of Mode S [D]. Chengdu: University of Electronic Science and Technology of China, 2012.

[10] Wang Fei. Research on ADS-B Key Techniques Based on 1090MHz ES Data Link [D]. Chengdu: University of Electronic Science and Technology of China, 2009.

[11] Jan Roskam. Airplane Design [M]. Roskam Aviation and Engineering Corporation, 1985.

[12] He Jiali; Liang Guozhu; Qiu Wei. Research on the Application of Aircraft Aerodynamic Calculation Method [J]. Aeronautical Computing Technique, 2008, 38(1): 47-51. 